\title{
INEQUALITIES FOR POWER SERIES WITH NONNEGATIVE COEFFICIENTS VIA A REVERSE OF JENSEN INEQUALITY
}

\author{
SILVESTRU SEVER DRAGOMIR
}

\begin{abstract}
Some inequalities for power series with nonnegative coefficients via a reverse of Jensen inequality obtained by Dragomir \& Ionescu in 1994 are given. Applications for some fundamental functions defined by power series are also provided.
\end{abstract}

\section{Introduction}

In 1994, Dragomir \& Ionescu obtained the following reverse of Jensen's discrete inequality:

Let $\Phi: I \rightarrow \mathbb{R}$ be a differentiable convex function on the interior $\stackrel{\circ}{I}$ of the interval $I$. If $x_{i} \in \stackrel{\circ}{I}$ and $w_{i} \geq 0(i=1, \ldots, n)$ with $W_{n}:=\sum_{i=1}^{n} w_{i}=1$, then one has the inequality:

$$
\begin{aligned}
0 & \leq \sum_{i=1}^{n} w_{i} \Phi\left(x_{i}\right)-\Phi\left(\sum_{i=1}^{n} w_{i} x_{i}\right) \\
& \leq \sum_{i=1}^{n} w_{i} \Phi^{\prime}\left(x_{i}\right) x_{i}-\sum_{i=1}^{n} w_{i} \Phi^{\prime}\left(x_{i}\right) \sum_{i=1}^{n} w_{i} x_{i} .
\end{aligned}
$$

In order to improve Grüss' discrete inequality, Cerone \& Dragomir established in 2002 the following result [1]:

$$
\begin{aligned}
\left|\sum_{i=1}^{n} w_{i} a_{i} b_{i}-\sum_{i=1}^{n} w_{i} a_{i} \sum_{i=1}^{n} w_{i} b_{i}\right| & \leq \frac{1}{2}(A-a) \sum_{i=1}^{n} w_{i}\left|b_{i}-\sum_{j=1}^{n} w_{j} b_{j}\right| \\
& \leq \frac{1}{2}(A-a)\left[\sum_{i=1}^{n} w_{i} b_{i}^{2}-\left(\sum_{i=1}^{n} w_{i} b_{i}\right)^{2}\right]^{1 / 2},
\end{aligned}
$$

provided $-\infty<a \leq a_{i} \leq A<\infty$, and $w_{i} \geq 0 \quad(i=1, \ldots, n)$ with $W_{n}:=\sum_{i=1}^{n} w_{i}=1$.

Received April 28, 2015, accepted June 1, 2015.

2010 Mathematics Subject Classification. 26D15; 26D10.

Key words and phrases. Power series, Jensen's inequality, reverse of Jensen's inequality. 
In addition, if $-\infty<b \leq b_{i} \leq B<\infty,(i=1, \ldots, n)$ then we have the string of inequalities

$$
\begin{aligned}
\left|\sum_{i=1}^{n} w_{i} a_{i} b_{i}-\sum_{i=1}^{n} w_{i} a_{i} \sum_{i=1}^{n} w_{i} b_{i}\right| & \leq \frac{1}{2}(A-a) \sum_{i=1}^{n} w_{i}\left|b_{i}-\sum_{j=1}^{n} w_{j} b_{j}\right| \\
& \leq \frac{1}{2}(A-a)\left[\sum_{i=1}^{n} w_{i} b_{i}^{2}-\left(\sum_{i=1}^{n} w_{i} b_{i}\right)^{2}\right]^{1 / 2} \\
& \leq \frac{1}{4}(A-a)(B-b) .
\end{aligned}
$$

Utilising these results, we observe that if $\Phi$ is differentiable convex on a finite interval, say $[m, M]$, then we have the inequalities:

$$
\begin{aligned}
0 & \leq \sum_{i=1}^{n} w_{i} \Phi\left(x_{i}\right)-\Phi\left(\sum_{i=1}^{n} w_{i} x_{i}\right) \\
& \leq \sum_{i=1}^{n} w_{i} \Phi^{\prime}\left(x_{i}\right) x_{i}-\sum_{i=1}^{n} w_{i} \Phi^{\prime}\left(x_{i}\right) \sum_{i=1}^{n} w_{i} x_{i} \\
& \leq \frac{1}{2}(M-m) \sum_{i=1}^{n} w_{i}\left|\Phi^{\prime}\left(x_{i}\right)-\sum_{j=1}^{n} w_{j} \Phi^{\prime}\left(x_{j}\right)\right| \\
& \leq \frac{1}{2}(M-m)\left[\sum_{i=1}^{n} w_{i}\left[\Phi^{\prime}\left(x_{i}\right)\right]^{2}-\left(\sum_{i=1}^{n} w_{i} \Phi^{\prime}\left(x_{i}\right)\right)^{2}\right]^{1 / 2}
\end{aligned}
$$

for $x_{i} \in(m, M)(i=1, \ldots, n)$.

If the lateral derivatives $\Phi_{+}^{\prime}(m)$ and $\Phi_{-}^{\prime}(M)$ are finite, then we also have

$$
\begin{aligned}
0 & \leq \sum_{i=1}^{n} w_{i} \Phi\left(x_{i}\right)-\Phi\left(\sum_{i=1}^{n} w_{i} x_{i}\right) \\
& \leq \sum_{i=1}^{n} w_{i} \Phi^{\prime}\left(x_{i}\right) x_{i}-\sum_{i=1}^{n} w_{i} \Phi^{\prime}\left(x_{i}\right) \sum_{i=1}^{n} w_{i} x_{i} \\
& \leq \frac{1}{2}\left[\Phi_{-}^{\prime}(M)-\Phi_{+}^{\prime}(m)\right] \sum_{i=1}^{n} w_{i}\left|x_{i}-\sum_{j=1}^{n} w_{j} x_{j}\right| \\
& \leq \frac{1}{2}\left[\Phi_{-}^{\prime}(M)-\Phi_{+}^{\prime}(m)\right]\left[\sum_{i=1}^{n} w_{i} x_{i}^{2}-\left(\sum_{i=1}^{n} w_{i} x_{i}\right)^{2}\right]^{1 / 2} \\
& \leq \frac{1}{4}(M-m)\left[\Phi_{-}^{\prime}(M)-\Phi_{+}^{\prime}(m)\right]
\end{aligned}
$$

for $x_{i} \in[m, M](i=1, \ldots, n)$.

In the recent paper [9], by the use of a refinement of Young's inequality, the authors proved the following result: 
Let $f(z)=\sum_{n=0}^{\infty} a_{n} z^{n}$ be a power series with nonnegative coefficients and convergent on the open disk $D(0, R)$ with $R>0$ or $R=\infty$. If $y, z, z^{v} y^{1-v}, z^{1-v} y^{v} \in(0, R)$ and $v \in[0,1]$ then we have the inequalities:

$$
\begin{aligned}
2 \min \{v, 1-v\}\left[f(y) f(z)-f^{2}(\sqrt{y z})\right] & \leq f(y) f(z)-f\left(z^{v} y^{1-v}\right) f\left(z^{1-v} y^{v}\right) \\
& \leq 2 \max \{v, 1-v\}\left[f(y) f(z)-f^{2}(\sqrt{y z})\right]
\end{aligned}
$$

or, equivalently,

$$
\begin{aligned}
2 \min \{v, 1-v\}\left[f\left(u^{2}\right) f\left(t^{2}\right)-f^{2}(u t)\right] & \leq f\left(u^{2}\right) f\left(t^{2}\right)-f\left(u^{2 v} t^{2(1-v)}\right) f\left(t^{2(1-v)} u^{2 v}\right) \\
& \leq 2 \max \{v, 1-v\}\left[f\left(u^{2}\right) f\left(t^{2}\right)-f^{2}(u t)\right],
\end{aligned}
$$

provided $u^{2}, t^{2}, u^{2 v} t^{2(1-v)}, t^{2(1-v)} u^{2 v} \in(0, R)$ and $v \in[0,1]$.

For other recent results for power series with nonnegative coefficients, see [9] and [10]. For more results on power series inequalities, see [2] and [5]-[8].

Motivated by the above results and utilizing a reverse of Jensen inequality obtained by Dragomir \& Ionescu in 1994 we provide in this paper other inequalities for power series with nonnegative coefficients. Applications for some fundamental and special functions are given as well.

\section{Power inequalities}

The most important power series with nonnegative coefficients are:

$$
\begin{aligned}
& \exp (z)=\sum_{n=0}^{\infty} \frac{1}{n !} z^{n}, z \in \mathbb{C}, \frac{1}{1-z}=\sum_{n=0}^{\infty} z^{n}, z \in D(0,1) \\
& \ln \frac{1}{1-z}=\sum_{n=1}^{\infty} \frac{1}{n} z^{n}, z \in D(0,1), \cosh z=\sum_{n=0}^{\infty} \frac{1}{(2 n) !} z^{2 n}, z \in \mathbb{C}, \\
& \sinh z=\sum_{n=0}^{\infty} \frac{1}{(2 n+1) !} z^{2 n+1}, z \in \mathbb{C} .
\end{aligned}
$$

Other important examples of functions as power series representations with nonnegative coefficients are:

$$
\begin{aligned}
\frac{1}{2} \ln \left(\frac{1+z}{1-z}\right) & =\sum_{n=1}^{\infty} \frac{1}{2 n-1} z^{2 n-1}, z \in D(0,1) \\
\sin ^{-1}(z) & =\sum_{n=0}^{\infty} \frac{\Gamma\left(n+\frac{1}{2}\right)}{\sqrt{\pi}(2 n+1) n !} z^{2 n+1}, z \in D(0,1), \\
\tanh ^{-1}(z) & =\sum_{n=1}^{\infty} \frac{1}{2 n-1} z^{2 n-1}, z \in D(0,1)
\end{aligned}
$$




$$
{ }_{2} F_{1}(\alpha, \beta, \gamma, z):=\sum_{n=0}^{\infty} \frac{\Gamma(n+\alpha) \Gamma(n+\beta) \Gamma(\gamma)}{n ! \Gamma(\alpha) \Gamma(\beta) \Gamma(n+\gamma)} z^{n}, \alpha, \beta, \gamma>0 \quad z \in D(0,1),
$$

where $\Gamma$ is Gamma function.

The following result for powers holds:

Theorem 1. Let $f(z)=\sum_{n=0}^{\infty} a_{n} z^{n}$ be a power series with nonnegative coefficients and convergent on the open disk $D(0, R)$ with $R>0$ or $R=\infty$. If $p \geq 1,0<\alpha<R$ and $x>0$ with $\alpha x^{p}, \alpha x^{p-1}<R$, then

$$
0 \leq \frac{f\left(\alpha x^{p}\right)}{f(\alpha)}-\left[\frac{f(\alpha x)}{f(\alpha)}\right]^{p} \leq p\left[\frac{f\left(\alpha x^{p}\right)}{f(\alpha)}-\frac{f\left(\alpha x^{p-1}\right)}{f(\alpha)} \frac{f(\alpha x)}{f(\alpha)}\right]
$$

Moreover, if $0<x \leq 1$, then

$$
\begin{aligned}
0 & \leq \frac{f\left(\alpha x^{p}\right)}{f(\alpha)}-\left[\frac{f(\alpha x)}{f(\alpha)}\right]^{p} \leq p\left[\frac{f\left(\alpha x^{p}\right)}{f(\alpha)}-\frac{f\left(\alpha x^{p-1}\right)}{f(\alpha)} \frac{f(\alpha x)}{f(\alpha)}\right] \\
& \leq \frac{1}{2} p\left(\frac{f\left(\alpha x^{2(p-1)}\right)}{f(\alpha)}-\left[\frac{f\left(\alpha x^{p-1}\right)}{f(\alpha)}\right]^{2}\right)^{1 / 2} \leq \frac{1}{4} p
\end{aligned}
$$

and

$$
\begin{aligned}
0 & \leq \frac{f\left(\alpha x^{p}\right)}{f(\alpha)}-\left[\frac{f(\alpha x)}{f(\alpha)}\right]^{p} \leq p\left[\frac{f\left(\alpha x^{p}\right)}{f(\alpha)}-\frac{f\left(\alpha x^{p-1}\right)}{f(\alpha)} \frac{f(\alpha x)}{f(\alpha)}\right] \\
& \leq \frac{1}{2} p\left(\frac{f\left(\alpha x^{2}\right)}{f(\alpha)}-\left[\frac{f(\alpha x)}{f(\alpha)}\right]^{2}\right)^{1 / 2} \leq \frac{1}{4} p .
\end{aligned}
$$

Proof. If we write the inequality (1.1) for the convex function $\Phi:[0, \infty) \rightarrow[0, \infty), \Phi(x)=x^{p}$, $p \geq 1$, then we have

$$
0 \leq \sum_{i=1}^{n} w_{i} x_{i}^{p}-\left(\sum_{i=1}^{n} w_{i} x_{i}\right)^{p} \leq p\left(\sum_{i=1}^{n} w_{i} x_{i}^{p}-\sum_{i=1}^{n} w_{i} x_{i}^{p-1} \sum_{i=1}^{n} w_{i} x_{i}\right)
$$

for any $w_{i}, x_{i} \geq 0(i=1, \ldots, n)$ with $\sum_{i=1}^{n} w_{i}=1$.

If $0<\alpha<R$ and $k \geq 1$, then by (2.6) we have

$$
\begin{aligned}
0 & \leq \frac{1}{\sum_{j=0}^{k} a_{j} \alpha^{j}} \sum_{j=0}^{k} a_{j} \alpha^{j}\left(x^{p}\right)^{j}-\left(\frac{1}{\sum_{j=0}^{k} a_{j} \alpha^{j}} \sum_{j=0}^{k} a_{j} \alpha^{j} x^{j}\right)^{p} \\
& \leq p\left[\frac{1}{\sum_{j=0}^{k} a_{j} \alpha^{j}} \sum_{j=0}^{k} a_{j} \alpha^{j}\left(x^{p}\right)^{j}-\frac{1}{\sum_{j=0}^{k} a_{j} \alpha^{j}} \sum_{j=0}^{k} a_{j} \alpha^{j}\left(x^{p-1}\right)^{j} \frac{1}{\sum_{j=0}^{k} a_{j} \alpha^{j}} \sum_{j=0}^{k} a_{j} \alpha^{j} x^{j}\right] .
\end{aligned}
$$

Since all series whose partial sums involved in the inequality (2.7) are convergent, then by letting $k \rightarrow \infty$ in (2.7) we deduce (2.3). 
Now, if $x_{i} \in[m, M] \subset[0, \infty),(i=1, \ldots, n)$, then by (1.4) for the convex function $\Phi:[0, \infty) \rightarrow$ $[0, \infty), \Phi(x)=x^{p}, p \geq 1$ we have

$$
\begin{aligned}
0 & \leq \sum_{i=1}^{n} w_{i} x_{i}^{p}-\left(\sum_{i=1}^{n} w_{i} x_{i}\right)^{p} \\
& \leq p\left(\sum_{i=1}^{n} w_{i} x_{i}^{p}-\sum_{i=1}^{n} w_{i} x_{i}^{p-1} \sum_{i=1}^{n} w_{i} x_{i}\right) \\
& \leq \frac{1}{2} p(M-m) \sum_{i=1}^{n} w_{i}\left|x_{i}^{p-1}-\sum_{j=1}^{n} w_{j} x_{j}^{p-1}\right| \\
& \leq \frac{1}{2} p(M-m)\left[\sum_{i=1}^{n} w_{i} x_{i}^{2(p-1)}-\left(\sum_{i=1}^{n} w_{i} x_{i}^{p-1}\right)^{2}\right]^{1 / 2} \\
& \leq \frac{1}{2} p(M-m)\left(M^{p-1}-m^{p-1}\right) .
\end{aligned}
$$

If $0<x \leq 1$, then $0<x^{j} \leq 1$ for $j=0, \ldots, k$ and by (2.8) we have

$$
\begin{aligned}
0 & \leq \frac{1}{\sum_{j=0}^{k} a_{j} \alpha^{j}} \sum_{j=0}^{k} a_{j} \alpha^{j}\left(x^{p}\right)^{j}-\left(\frac{1}{\sum_{j=0}^{k} a_{j} \alpha^{j}} \sum_{j=0}^{k} a_{j} \alpha^{j} x^{j}\right)^{p} \\
& \leq p\left[\frac{1}{\sum_{j=0}^{k} a_{j} \alpha^{j}} \sum_{j=0}^{k} a_{j} \alpha^{j}\left(x^{p}\right)^{j}-\frac{1}{\sum_{j=0}^{k} a_{j} \alpha^{j}} \sum_{j=0}^{k} a_{j} \alpha^{j}\left(x^{p-1}\right)^{j} \frac{1}{\sum_{j=0}^{k} a_{j} \alpha^{j}} \sum_{j=0}^{k} a_{j} \alpha^{j} x^{j}\right] \\
& \leq \frac{1}{2} p\left[\frac{1}{\sum_{j=0}^{k} a_{j} \alpha^{j}} \sum_{j=0}^{k} a_{j} \alpha^{j}\left[x^{2(p-1)}\right]^{j}-\left(\frac{1}{\sum_{j=0}^{k} a_{j} \alpha^{j}} \sum_{j=0}^{k} a_{j} \alpha^{j}\left(x^{p-1}\right)^{j}\right)^{2}\right]^{1 / 2} \\
& \leq \frac{1}{4} p .
\end{aligned}
$$

Since all series whose partial sums involved in the inequality (2.9) are convergent, then by letting $k \rightarrow \infty$ in (2.9) we deduce (2.4).

Now, if $x_{i} \in[m, M] \subset[0, \infty),(i=1, \ldots, n)$, then by (1.5) for the convex function $\Phi:[0, \infty) \rightarrow$ $[0, \infty), \Phi(x)=x^{p}, p \geq 1$ we have

$$
\begin{aligned}
0 & \leq \sum_{i=1}^{n} w_{i} x_{i}^{p}-\left(\sum_{i=1}^{n} w_{i} x_{i}\right)^{p} \\
& \leq p\left(\sum_{i=1}^{n} w_{i} x_{i}^{p}-\sum_{i=1}^{n} w_{i} x_{i}^{p-1} \sum_{i=1}^{n} w_{i} x_{i}\right) \\
& \leq \frac{1}{2} p\left(M^{p-1}-m^{p-1}\right) \sum_{i=1}^{n} w_{i}\left|x_{i}-\sum_{j=1}^{n} w_{j} x_{j}\right| \\
& \leq \frac{1}{2} p\left(M^{p-1}-m^{p-1}\right)\left[\sum_{i=1}^{n} w_{i} x_{i}^{2}-\left(\sum_{i=1}^{n} w_{i} x_{i}\right)^{2}\right]^{1 / 2}
\end{aligned}
$$




$$
\leq \frac{1}{4}(M-m)\left(M^{p-1}-m^{p-1}\right)
$$

Finally, by utilizing a similar argument as above, we obtain the inequality (2.5). The details are omitted.

Remark 1. We observe that, the second inequality in (2.3) is equivalent to

or to

$$
\frac{f(\alpha x)}{f(\alpha)}\left(p \frac{f\left(\alpha x^{p-1}\right)}{f(\alpha)}-\left[\frac{f(\alpha x)}{f(\alpha)}\right]^{p-1}\right) \leq(p-1) \frac{f\left(\alpha x^{p}\right)}{f(\alpha)}
$$

$$
f(\alpha x)\left(p f\left(\alpha x^{p-1}\right)[f(\alpha)]^{p-2}-[f(\alpha x)]^{p-1}\right) \leq(p-1) f\left(\alpha x^{p}\right)[f(\alpha)]^{p-1},
$$

provided that $p \geq 1,0<\alpha<R$ and $x>0$ with $\alpha x^{p}, \alpha x^{p-1}<R$.

Moreover, if $0<x \leq 1$, then from (2.4) we have

$$
\left[\frac{f(\alpha x)}{f(\alpha)}\right]^{p} \leq \frac{f\left(\alpha x^{p}\right)}{f(\alpha)} \leq \frac{1}{4} p+\left[\frac{f(\alpha x)}{f(\alpha)}\right]^{p} .
$$

Taking the power $1 / p$ and using the inequality $(a+b)^{1 / p} \leq a^{1 / p}+b^{1 / p}, p \geq 1$ we get

$$
0 \leq\left[f\left(\alpha x^{p}\right)\right]^{1 / p}[f(\alpha)]^{1-\frac{1}{p}}-f(\alpha x) \leq \frac{1}{4^{1 / p}} p^{1 / p} f(\alpha) .
$$

Corollary 1. Let $f(z)=\sum_{n=0}^{\infty} a_{n} z^{n}$ be a power series with nonnegative coefficients and convergent on the open disk $D(0, R)$ with $R>0$ or $R=\infty$. If $p>1, \frac{1}{p}+\frac{1}{q}=1$ and $u, v>0$ with $v^{p} \leq u^{q}<R$, then

$$
\left[\frac{f(u v)}{f\left(u^{q}\right)}\right]^{p} \leq \frac{f\left(v^{p}\right)}{f\left(u^{q}\right)} \leq \frac{1}{4} p+\left[\frac{f(u v)}{f\left(u^{q}\right)}\right]^{p}
$$

and

$$
0 \leq\left[f\left(v^{p}\right)\right]^{1 / p}\left[f\left(u^{q}\right)\right]^{1 / q}-f(u v) \leq \frac{1}{4^{1 / p}} p^{1 / p} f\left(u^{q}\right)
$$

Proof. Follows by taking into (2.13) and (2.14) $\alpha=u^{q}$ and $x=\frac{v}{u^{q / p}}$. The details are omitted.

Example 1. a) If we write the inequalities (2.4) and (2.5) for the function $\frac{1}{1-z}=\sum_{n=0}^{\infty} z^{n}, z \in$ $D(0,1)$, then we have

$$
\begin{aligned}
0 & \leq \frac{1-\alpha}{1-\alpha x^{p}}-\left(\frac{1-\alpha}{1-\alpha x}\right)^{p} \leq p\left[\frac{1-\alpha}{1-\alpha x^{p}}-\frac{(1-\alpha)^{2}}{\left(1-\alpha x^{p-1}\right)(1-\alpha x)}\right] \\
& \leq \frac{1}{2} p\left[\frac{1-\alpha}{1-\alpha x^{2(p-1)}}-\left(\frac{1-\alpha}{1-\alpha x^{p-1}}\right)^{2}\right]^{1 / 2} \leq \frac{1}{4} p
\end{aligned}
$$

and

$$
0 \leq \frac{1-\alpha}{1-\alpha x^{p}}-\left(\frac{1-\alpha}{1-\alpha x}\right)^{p} \leq p\left[\frac{1-\alpha}{1-\alpha x^{p}}-\frac{(1-\alpha)^{2}}{\left(1-\alpha x^{p-1}\right)(1-\alpha x)}\right]
$$




$$
\leq \frac{1}{2} p\left[\frac{1-\alpha}{1-\alpha x^{2}}-\left(\frac{1-\alpha}{1-\alpha x}\right)^{2}\right]^{1 / 2} \leq \frac{1}{4} p
$$

for any $\alpha, x \in(0,1)$ and $p \geq 1$.

b) If we write the inequalities (2.4) and (2.5) for the function $\exp z=\sum_{n=0}^{\infty} \frac{1}{n !} z^{n}, z \in \mathbb{C}$, then we have

$$
\begin{aligned}
0 & \leq \exp \left[\alpha\left(x^{p}-1\right)\right]-\exp [p \alpha(x-1)] \\
& \leq p\left[\exp \left[\alpha\left(x^{p}-1\right)\right]-\exp \left[\alpha\left(x^{p-1}+x-2\right)\right]\right] \\
& \leq \frac{1}{2} p\left(\exp \left[\alpha\left(x^{2(p-1)}-1\right)\right]-\exp \left[2 \alpha\left(x^{p-1}-1\right)\right]\right)^{1 / 2} \leq \frac{1}{4} p
\end{aligned}
$$

and

$$
\begin{aligned}
0 & \leq \exp \left[\alpha\left(x^{p}-1\right)\right]-\exp [p \alpha(x-1)] \\
& \leq p\left[\exp \left[\alpha\left(x^{p}-1\right)\right]-\exp \left[\alpha\left(x^{p-1}+x-2\right)\right]\right] \\
& \leq \frac{1}{2} p\left(\exp \left[\alpha\left(x^{2}-1\right)\right]-\exp [2 \alpha(x-1)]\right)^{1 / 2} \leq \frac{1}{4} p .
\end{aligned}
$$

for any $\alpha, p>0$ and $x \in(0,1)$.

\section{Exponential inequalities}

The following exponential inequality holds:

Theorem 2. Let $f(z)=\sum_{n=0}^{\infty} a_{n} z^{n}$ be a power series with nonnegative coefficients and convergent on the open disk $D(0, R)$ with $R>0$ or $R=\infty$. If $0<\alpha<R$ and $x, \beta \in \mathbb{R}$ with $\alpha \exp (\beta x)<R$ then

$$
\begin{aligned}
0 & \leq \frac{f(\alpha \exp (\beta x))}{f(\alpha)}-\exp \left[\frac{\alpha \beta x f^{\prime}(\alpha)}{f(\alpha)}\right] \\
& \leq \alpha \beta x\left[\frac{\exp (\beta x) f^{\prime}(\alpha \exp (\beta x))}{f(\alpha)}-\frac{f(\alpha \exp (\beta x))}{f(\alpha)} \frac{f^{\prime}(\alpha)}{f(\alpha)}\right] .
\end{aligned}
$$

Moreover, if $x \leq 0, \beta>0$ with $\exp (\beta x)<R$ and $0<\alpha<R$, then

$$
\begin{aligned}
0 & \leq \frac{f(\alpha \exp (\beta x))}{f(\alpha)}-\exp \left[\frac{\alpha \beta x f^{\prime}(\alpha)}{f(\alpha)}\right] \\
& \leq \alpha \beta x\left[\frac{\exp (\beta x) f^{\prime}(\alpha \exp (\beta x))}{f(\alpha)}-\frac{f(\alpha \exp (\beta x))}{f(\alpha)} \frac{f^{\prime}(\alpha)}{f(\alpha)}\right] \\
& \leq \frac{1}{2} \beta|x|\left[\frac{\alpha\left[f^{\prime}(\alpha)+\alpha f^{\prime \prime}(\alpha)\right]}{f(\alpha)}-\left(\frac{\alpha f^{\prime}(\alpha)}{f(\alpha)}\right)^{2}\right]^{1 / 2} .
\end{aligned}
$$


Proof. If we write the inequality (1.1) for the convex function $\Phi: \mathbb{R} \rightarrow[0, \infty), \Phi(x)=\exp (\beta x)$, then we have

$$
\begin{aligned}
0 & \leq \sum_{i=1}^{n} w_{i} \exp \left(\beta x_{i}\right)-\exp \left(\beta \sum_{i=1}^{n} w_{i} x_{i}\right) \\
& \leq \beta\left[\sum_{i=1}^{n} w_{i} x_{i} \exp \left(\beta x_{i}\right)-\sum_{i=1}^{n} w_{i} \exp \left(\beta x_{i}\right) \sum_{i=1}^{n} w_{i} x_{i}\right]
\end{aligned}
$$

for any $w_{i} \geq 0(i=1, \ldots, n)$ with $\sum_{i=1}^{n} w_{i}=1$ and $x_{i} \in \mathbb{R}(i=1, \ldots, n)$.

If $0<\alpha<R$ and $k \geq 1$, then by (3.3) for $x_{j}=j x$, we have

$$
\begin{aligned}
0 \leq & \frac{1}{\sum_{j=0}^{k} a_{j} \alpha^{j}} \sum_{j=0}^{k} a_{j} \alpha^{j}[\exp (\beta x)]^{j}-\exp \left(\frac{\beta x}{\sum_{j=0}^{k} a_{j} \alpha^{j}} \sum_{j=0}^{k} j a_{j} \alpha^{j}\right) \\
\leq & \beta x\left[\frac{1}{\sum_{j=0}^{k} a_{j} \alpha^{j}} \sum_{j=0}^{k} j a_{j} \alpha^{j}[\exp (\beta x)]^{j}\right. \\
& \left.-\frac{1}{\sum_{j=0}^{k} a_{j} \alpha^{j}} \sum_{j=0}^{k} a_{j} \alpha^{j}[\exp (\beta x)]^{j} \frac{1}{\sum_{j=0}^{k} a_{j} \alpha^{j}} \sum_{j=0}^{k} j a_{j} \alpha^{j}\right] .
\end{aligned}
$$

Observe that the series $\sum_{j=0}^{\infty} j a_{j} \alpha^{j}$ is convergent for $0<\alpha<R$ and

$$
\sum_{j=0}^{\infty} j a_{j} \alpha^{j}=\sum_{j=1}^{\infty} j a_{j} \alpha^{j}=\alpha f^{\prime}(\alpha), 0<\alpha<R .
$$

Since all series whose partial sums involved in the inequality (3.4) are convergent, then by letting $k \rightarrow \infty$ in (3.4) we deduce (3.1).

If we write the inequality (1.4) for the convex function $\Phi: \mathbb{R} \rightarrow[0, \infty), \Phi(x)=\exp (\beta x)$, then we have

$$
\begin{aligned}
0 & \leq \sum_{i=1}^{n} w_{i} \exp \left(\beta x_{i}\right)-\exp \left(\beta \sum_{i=1}^{n} w_{i} x_{i}\right) \\
& \leq \beta\left[\sum_{i=1}^{n} w_{i} x_{i} \exp \left(\beta x_{i}\right)-\sum_{i=1}^{n} w_{i} \exp \left(\beta x_{i}\right) \sum_{i=1}^{n} w_{i} x_{i}\right] \\
& \leq \frac{1}{2} \beta[\exp (\beta M)-\exp (\beta m)] \sum_{i=1}^{n} w_{i}\left|x_{i}-\sum_{j=1}^{n} w_{j} x_{j}\right| \\
& \leq \frac{1}{2} \beta[\exp (\beta M)-\exp (\beta m)]\left[\sum_{i=1}^{n} w_{i} x_{i}^{2}-\left(\sum_{i=1}^{n} w_{i} x_{i}\right)^{2}\right]^{1 / 2},
\end{aligned}
$$

provided $m \leq x_{i} \leq M, i \in\{1, \ldots, n\}$.

Now, if $\beta>0$, by letting $M=0$ and $m \rightarrow-\infty$ then by (3.5) we have

$$
0 \leq \sum_{i=1}^{n} w_{i} \exp \left(\beta x_{i}\right)-\exp \left(\beta \sum_{i=1}^{n} w_{i} x_{i}\right)
$$




$$
\begin{aligned}
& \leq \beta\left[\sum_{i=1}^{n} w_{i} x_{i} \exp \left(\beta x_{i}\right)-\sum_{i=1}^{n} w_{i} \exp \left(\beta x_{i}\right) \sum_{i=1}^{n} w_{i} x_{i}\right] \\
& \leq \frac{1}{2} \beta \sum_{i=1}^{n} w_{i}\left|x_{i}-\sum_{j=1}^{n} w_{j} x_{j}\right| \leq \frac{1}{2} \beta\left[\sum_{i=1}^{n} w_{i} x_{i}^{2}-\left(\sum_{i=1}^{n} w_{i} x_{i}\right)^{2}\right]^{1 / 2},
\end{aligned}
$$

provided $-\infty<x_{i} \leq 0$.

If $0<\alpha<R, x \leq 0, \beta>0$ and $k \geq 1$, then by (3.3) for $x_{j}=j x \in(-\infty, 0]$, we have

$$
\begin{aligned}
0 \leq & \frac{1}{\sum_{j=0}^{k} a_{j} \alpha^{j}} \sum_{j=0}^{k} a_{j} \alpha^{j}[\exp (\beta x)]^{j}-\exp \left(\frac{\beta x}{\sum_{j=0}^{k} a_{j} \alpha^{j}} \sum_{j=0}^{k} j a_{j} \alpha^{j}\right) \\
\leq & \beta x\left[\frac{1}{\sum_{j=0}^{k} a_{j} \alpha^{j}} \sum_{j=0}^{k} j a_{j} \alpha^{j}[\exp (\beta x)]^{j}\right. \\
& \left.-\frac{1}{\sum_{j=0}^{k} a_{j} \alpha^{j}} \sum_{j=0}^{k} a_{j} \alpha^{j}[\exp (\beta x)]^{j} \frac{1}{\sum_{j=0}^{k} a_{j} \alpha^{j}} \sum_{j=0}^{k} j a_{j} \alpha^{j}\right] \\
\leq & \frac{1}{2} \beta|x|\left[\frac{1}{\sum_{j=0}^{k} a_{j} \alpha^{j}} \sum_{j=0}^{k} j^{2} a_{j} \alpha^{j}-\left(\frac{1}{\sum_{j=0}^{k} a_{j} \alpha^{j}} \sum_{j=0}^{k} j a_{j} \alpha^{j}\right)^{2}\right]^{1 / 2} .
\end{aligned}
$$

If we denote $g(u):=\sum_{n=0}^{\infty} \alpha_{n} u^{n}$, then for $|u|<R$, its radius of convergence, we have

$$
\sum_{n=0}^{\infty} n \alpha_{n} u^{n}=u g^{\prime}(u)
$$

and

$$
\sum_{n=0}^{\infty} n^{2} \alpha_{n} u^{n}=u\left(u g^{\prime}(u)\right)^{\prime}
$$

However

$$
u\left(u g^{\prime}(u)\right)^{\prime}=u g^{\prime}(u)+u^{2} g^{\prime \prime}(u)
$$

and then

$$
\sum_{n=0}^{\infty} n^{2} \alpha_{n} u^{n}=u g^{\prime}(u)+u^{2} g^{\prime \prime}(u) .
$$

Since all series whose partial sums involved in the inequality (3.7) are convergent, then by letting $k \rightarrow \infty$ in (3.7) we deduce (3.2).

\section{Example 2.}

(a) If we write the inequality (3.2) for the function $\frac{1}{1-z}=\sum_{n=0}^{\infty} z^{n}, z \in D(0,1)$, then we have for $x \leq 0, \beta>0$ and $0<\alpha<1$, that

$$
\begin{aligned}
0 & \leq \frac{1-\alpha}{1-\alpha \exp (\beta x)}-\exp \left(\frac{\alpha \beta x}{1-\alpha}\right) \\
& \leq \alpha \beta x\left[\frac{(1-\alpha) \exp (\beta x)}{(1-\alpha \exp (\beta x))^{2}}-\frac{1}{1-\alpha \exp (\beta x)}\right] \leq \frac{1}{2} \frac{\beta|x| \alpha^{1 / 2}}{1-\alpha} .
\end{aligned}
$$


(b) If we write the inequality (3.1) for the function $\exp z=\sum_{n=0}^{\infty} \frac{1}{n !} z^{n}, z \in \mathbb{C}$, then we have

$$
\begin{aligned}
0 & \leq \exp (\alpha[\exp (\beta x)-1])-\exp (\alpha \beta x) \\
& \leq \alpha \beta x[\exp (\alpha[\exp (\beta x)-1]+\beta x)-\exp (\alpha[\exp (\beta x)-1])]
\end{aligned}
$$

for any $\alpha>0$ and $x \leq 0, \beta>0$.

\subsection{Logarithmic Inequalities}

The following logarithmic inequality holds:

Theorem 3. Let $f(z)=\sum_{n=0}^{\infty} a_{n} z^{n}$ be a power series with nonnegative coefficients and convergent on the open disk $D(0, R)$ with $R>0$ or $R=\infty$. If $0<\alpha<R, p>0$ and $x>0$ with $\alpha x^{p}, \alpha x^{-p}<R$, then

$$
0 \leq \ln \left(\frac{f\left(\alpha x^{p}\right)}{f(\alpha)}\right)-p \frac{\alpha f^{\prime}(\alpha)}{f(\alpha)} \ln x \leq \frac{f\left(\alpha x^{p}\right)}{f(\alpha)} \frac{f\left(\alpha x^{-p}\right)}{f(\alpha)}-1 .
$$

Moreover, if $0<x \leq 1$ with $\alpha x^{p}, \alpha x^{-p}<R$, then

$$
\begin{aligned}
0 & \leq \ln \left(\frac{f\left(\alpha x^{p}\right)}{f(\alpha)}\right)-p \frac{\alpha f^{\prime}(\alpha)}{f(\alpha)} \ln x \leq \frac{f\left(\alpha x^{p}\right)}{f(\alpha)} \frac{f\left(\alpha x^{-p}\right)}{f(\alpha)}-1 \\
& \leq \frac{1}{2}\left[\frac{f\left(\alpha x^{-2 p}\right)}{f(\alpha)}-\left(\frac{f\left(\alpha x^{-p}\right)}{f(\alpha)}\right)^{2}\right]^{1 / 2} .
\end{aligned}
$$

Proof. If we write the inequality (1.1) for the convex function $\Phi:(0, \infty) \rightarrow \mathbb{R}, \Phi(x)=-\ln x$, then we have

$$
0 \leq \ln \left(\sum_{i=1}^{n} w_{i} x_{i}\right)-\sum_{i=1}^{n} w_{i} \ln \left(x_{i}\right) \leq \sum_{i=1}^{n} \frac{w_{i}}{x_{i}} \sum_{i=1}^{n} w_{i} x_{i}-1,
$$

for any $w_{i} \geq 0(i=1, \ldots, n)$ with $\sum_{i=1}^{n} w_{i}=1$ and $x_{i} \in(0, \infty)(i=1, \ldots, n)$.

If $0<\alpha<R$ and $k \geq 1$, then by (3.12) for $x_{j}=\left(x^{p}\right)^{j}$, we have

$$
\begin{aligned}
0 & \leq \ln \left(\frac{1}{\sum_{j=0}^{k} a_{j} \alpha^{j}} \sum_{j=0}^{k} a_{j} \alpha^{j}\left(x^{p}\right)^{j}\right)-\frac{p \ln x}{\sum_{j=0}^{k} a_{j} \alpha^{j}} \sum_{j=0}^{k} j a_{j} \alpha^{j} \\
& \leq \frac{1}{\sum_{j=0}^{k} a_{j} \alpha^{j}} \sum_{j=0}^{k} a_{j} \alpha^{j}\left(x^{p}\right)^{j} \frac{1}{\sum_{j=0}^{k} a_{j} \alpha^{j}} \sum_{j=0}^{k} a_{j} \frac{\alpha^{j}}{\left(x^{p}\right)^{j}}-1 .
\end{aligned}
$$

Since all series whose partial sums involved in the inequality (3.13) are convergent, then by letting $k \rightarrow \infty$ in (3.13) we deduce (3.10).

From the inequality (1.4) we have

$$
0 \leq \ln \left(\sum_{i=1}^{n} w_{i} x_{i}\right)-\sum_{i=1}^{n} w_{i} \ln \left(x_{i}\right) \leq \sum_{i=1}^{n} \frac{w_{i}}{x_{i}} \sum_{i=1}^{n} w_{i} x_{i}-1
$$




$$
\begin{aligned}
& \leq \frac{1}{2}(M-m) \sum_{i=1}^{n} w_{i}\left|\frac{1}{x_{i}}-\sum_{j=1}^{n} \frac{w_{j}}{x_{j}}\right| \\
& \leq \frac{1}{2}(M-m)\left[\sum_{i=1}^{n} \frac{w_{i}}{x_{i}^{2}}-\left(\sum_{i=1}^{n} \frac{w_{i}}{x_{i}}\right)^{2}\right]^{1 / 2}
\end{aligned}
$$

for any $w_{i} \geq 0(i=1, \ldots, n)$ with $\sum_{i=1}^{n} w_{i}=1$ and $x_{i} \in[m, M] \subset(0, \infty)(i=1, \ldots, n)$.

If $0<x \leq 1$, then $0<x^{p} \leq 1$ and if we apply the inequality (3.14) for $x_{j}=\left(x^{p}\right)^{j} \in(0,1]$ we have

$$
\begin{aligned}
0 & \leq \ln \left(\frac{1}{\sum_{j=0}^{k} a_{j} \alpha^{j}} \sum_{j=0}^{k} a_{j} \alpha^{j}\left(x^{p}\right)^{j}\right)-\frac{p \ln x}{\sum_{j=0}^{k} a_{j} \alpha^{j}} \sum_{j=0}^{k} j a_{j} \alpha^{j} \\
& \leq \frac{1}{\sum_{j=0}^{k} a_{j} \alpha^{j}} \sum_{j=0}^{k} a_{j} \alpha^{j}\left(x^{p}\right)^{j} \frac{1}{\sum_{j=0}^{k} a_{j} \alpha^{j}} \sum_{j=0}^{k} a_{j} \frac{\alpha^{j}}{\left(x^{p}\right)^{j}}-1 \\
& \leq \frac{1}{2}\left[\frac{1}{\sum_{j=0}^{k} a_{j} \alpha^{j}} \sum_{j=0}^{k} a_{j} \frac{\alpha^{j}}{\left(x^{2 p}\right)^{j}}-\left(\frac{1}{\sum_{j=0}^{k} a_{j} \alpha^{j}} \sum_{j=0}^{k} a_{j} \frac{\alpha^{j}}{\left(x^{p}\right)^{j}}\right)^{2}\right]^{1 / 2} .
\end{aligned}
$$

Since all series whose partial sums involved in the inequality (3.15) are convergent, then by letting $k \rightarrow \infty$ in (3.15) we deduce (3.11).

Corollary 2. Let $f(z)=\sum_{n=0}^{\infty} a_{n} z^{n}$ be a power series with nonnegative coefficients and convergent on the open disk $D(0, R)$ with $R>0$ or $R=\infty$. If $p>1, \frac{1}{p}+\frac{1}{q}=1$ and $u, v>0$ with $v^{p}, u^{q}, \frac{u^{2 q}}{v^{p}}<R$, then

$$
0 \leq \ln \left(\frac{f\left(v^{p}\right)}{f\left(u^{q}\right)}\right)-\frac{u^{q} f^{\prime}\left(u^{q}\right)}{f\left(u^{q}\right)} \ln \frac{v^{p}}{u^{q}} \leq \frac{f\left(v^{p}\right)}{f\left(u^{q}\right)} \frac{f\left(\frac{u^{2 q}}{v^{p}}\right)}{f\left(u^{q}\right)}-1 .
$$

If $v^{p} \leq u^{q}<R$ and $\frac{u^{2 q}}{v^{p}}, \frac{u^{3 q}}{v^{2 p}}$, then

$$
\begin{aligned}
0 & \leq \ln \left(\frac{f\left(v^{p}\right)}{f\left(u^{q}\right)}\right)-\frac{u^{q} f^{\prime}\left(u^{q}\right)}{f\left(u^{q}\right)} \ln \frac{v^{p}}{u^{q}} \leq \frac{f\left(v^{p}\right)}{f\left(u^{q}\right)} \frac{f\left(\frac{u^{2 q}}{v^{p}}\right)}{f\left(u^{q}\right)}-1 \\
& \leq \frac{1}{2}\left[\frac{f\left(\frac{u^{3 q}}{v^{2 p}}\right)}{f\left(u^{q}\right)}-\left(\frac{f\left(\frac{u^{2 q}}{v^{p}}\right)}{f\left(u^{q}\right)}\right)^{2}\right]^{1 / 2} .
\end{aligned}
$$

Proof. Follows by taking into (3.10) and (3.11) $\alpha=u^{q}$ and $x=\frac{v}{u^{q / p}}$. The details are omitted.

\section{Example 3.}

(a) If we write the inequality (3.11) for the function $\frac{1}{1-z}=\sum_{n=0}^{\infty} z^{n}, z \in D(0,1)$, then we have for $0<\alpha, x<1$ and $p>0$ that

$$
0 \leq \ln \left(\frac{1-\alpha}{1-\alpha x^{p}}\right)-\frac{p \alpha}{1-\alpha} \ln x \leq \frac{(1-\alpha)^{2}}{\left(1-\alpha x^{p}\right)\left(1-\alpha x^{-p}\right)}-1
$$




$$
\leq \frac{1}{2}\left[\frac{1-\alpha}{1-\alpha x^{-2 p}}-\left(\frac{1-\alpha}{1-\alpha x^{-p}}\right)^{2}\right]^{1 / 2}
$$

(b) If we write the inequality (3.10) for the function $\exp z=\sum_{n=0}^{\infty} \frac{1}{n !} z^{n}, z \in \mathbb{C}$, then we have

$$
0 \leq \alpha\left(x^{p}-1\right)-p \alpha \ln x \leq \exp \left[\alpha\left(x^{p}+x^{-p}-2\right)\right]-1
$$

for $\alpha, p, x>0$.

The following logarithmic inequality also holds:

Theorem 4. Let $f(z)=\sum_{n=0}^{\infty} a_{n} z^{n}$ be a power series with nonnegative coefficients and convergent on the open disk $D(0, R)$ with $R>0$ or $R=\infty$. If $0<\alpha<R, p>0$ and $x>0$ with $\alpha x^{p}<R$, then

$$
\begin{aligned}
0 & \leq \frac{p \alpha x^{p} f^{\prime}\left(\alpha x^{p}\right)}{f(\alpha)} \ln x-\frac{f\left(\alpha x^{p}\right)}{f(\alpha)} \ln \left(\frac{f\left(\alpha x^{p}\right)}{f(\alpha)}\right) \\
& \leq p \alpha\left[\frac{x^{p} f^{\prime}\left(\alpha x^{p}\right)}{f(\alpha)}-\frac{f\left(\alpha x^{p}\right)}{f(\alpha)} \frac{f^{\prime}(\alpha)}{f(\alpha)}\right] \ln x,
\end{aligned}
$$

or, equivalently

$$
\frac{p \alpha f^{\prime}(\alpha)}{f(\alpha)} \ln x \leq \ln \left(\frac{f\left(\alpha x^{p}\right)}{f(\alpha)}\right)
$$

i.e., the first inequality in (3.10).

Moreover, if $0<x \leq 1$ we also have

$$
\begin{aligned}
0 & \leq \frac{p \alpha x^{p} f^{\prime}\left(\alpha x^{p}\right)}{f(\alpha)} \ln x-\frac{f\left(\alpha x^{p}\right)}{f(\alpha)} \ln \left(\frac{f\left(\alpha x^{p}\right)}{f(\alpha)}\right) \\
& \leq p \alpha\left[\frac{x^{p} f^{\prime}\left(\alpha x^{p}\right)}{f(\alpha)}-\frac{f\left(\alpha x^{p}\right)}{f(\alpha)} \frac{f^{\prime}(\alpha)}{f(\alpha)}\right] \ln x \\
& \leq \frac{1}{2} p|\ln x|\left[\frac{\alpha\left[f^{\prime}(\alpha)+\alpha f^{\prime \prime}(\alpha)\right]}{f(\alpha)}-\left(\frac{\alpha f^{\prime}(\alpha)}{f(\alpha)}\right)^{2}\right]^{1 / 2} .
\end{aligned}
$$

Proof. If we write the inequality (1.1) for the convex function $\Phi:(0, \infty) \rightarrow \mathbb{R}, \Phi(x)=x \ln x$, then we have

$$
\begin{aligned}
0 & \leq \sum_{i=1}^{n} w_{i} x_{i} \ln \left(x_{i}\right)-\sum_{i=1}^{n} w_{i} x_{i} \ln \left(\sum_{i=1}^{n} w_{i} x_{i}\right) \\
& \leq \sum_{i=1}^{n} w_{i}\left[\ln \left(x_{i}\right)+1\right] x_{i}-\sum_{i=1}^{n} w_{i}\left[\ln \left(x_{i}\right)+1\right] \sum_{i=1}^{n} w_{i} x_{i}
\end{aligned}
$$

for any $w_{i} \geq 0(i=1, \ldots, n)$ with $\sum_{i=1}^{n} w_{i}=1$ and $x_{i} \in(0, \infty)(i=1, \ldots, n)$. 
If $0<\alpha<R$ and $k \geq 1$, then by (3.22) for $x_{j}=\left(x^{p}\right)^{j}$, we have

$$
\begin{aligned}
0 & \leq \frac{p \ln x}{\sum_{j=0}^{k} a_{j} \alpha^{j}} \sum_{j=0}^{k} j a_{j} \alpha^{j}\left(x^{p}\right)^{j} \\
& -\frac{1}{\sum_{j=0}^{k} a_{j} \alpha^{j}} \sum_{j=0}^{k} a_{j} \alpha^{j}\left(x^{p}\right)^{j} \ln \left(\frac{1}{\sum_{j=0}^{k} a_{j} \alpha^{j}} \sum_{j=0}^{k} a_{j} \alpha^{j}\left(x^{p}\right)^{j}\right) \\
& \leq \frac{1}{\sum_{j=0}^{k} a_{j} \alpha^{j}} \sum_{j=0}^{k} a_{j} \alpha^{j}(j p \ln x+1)\left(x^{p}\right)^{j} \\
& -\frac{1}{\sum_{j=0}^{k} a_{j} \alpha^{j}} \sum_{j=0}^{k} a_{j} \alpha^{j}(j p \ln x+1) \frac{1}{\sum_{j=0}^{k} a_{j} \alpha^{j}} \sum_{j=0}^{k} a_{j} \alpha^{j}\left(x^{p}\right)^{j} \\
& =\frac{1}{\sum_{j=0}^{k} a_{j} \alpha^{j}}\left[p \ln x \sum_{j=0}^{k} j a_{j} \alpha^{j}\left(x^{p}\right)^{j}+\sum_{j=0}^{k} a_{j} \alpha^{j}\left(x^{p}\right)^{j}\right] \\
& -\frac{1}{\sum_{j=0}^{k} a_{j} \alpha^{j}}\left[p \ln x \sum_{j=0}^{k} j a_{j} \alpha^{j}+\sum_{j=0}^{k} a_{j} \alpha^{j}\right] \frac{1}{\sum_{j=0}^{k} a_{j} \alpha^{j}} \sum_{j=0}^{k} a_{j} \alpha^{j}\left(x^{p}\right)^{j} .
\end{aligned}
$$

Since all series whose partial sums involved in the inequality (3.23) are convergent, then by letting $k \rightarrow \infty$ in (3.23) we deduce

$$
\begin{aligned}
0 & \leq \frac{p \alpha x^{p} f^{\prime}\left(\alpha x^{p}\right)}{f(\alpha)} \ln x-\frac{f\left(\alpha x^{p}\right)}{f(\alpha)} \ln \left(\frac{f\left(\alpha x^{p}\right)}{f(\alpha)}\right) \\
& \leq \frac{1}{f(\alpha)}\left[p \alpha x^{p} f^{\prime}\left(\alpha x^{p}\right) \ln x+f\left(\alpha x^{p}\right)\right]-\frac{1}{f(\alpha)}\left[p \alpha f^{\prime}(\alpha) \ln x+f(\alpha)\right] \frac{f\left(\alpha x^{p}\right)}{f(\alpha)},
\end{aligned}
$$

which is equivalent to (3.20).

If we write the inequality (1.4) for the convex function $\Phi:(0, \infty) \rightarrow \mathbb{R}, \Phi(x)=x \ln x$, then we have

$$
\begin{aligned}
0 & \leq \sum_{i=1}^{n} w_{i} x_{i} \ln \left(x_{i}\right)-\sum_{i=1}^{n} w_{i} x_{i} \ln \left(\sum_{i=1}^{n} w_{i} x_{i}\right) \\
& \leq \sum_{i=1}^{n} w_{i}\left[\ln \left(x_{i}\right)+1\right] x_{i}-\sum_{i=1}^{n} w_{i}\left[\ln \left(x_{i}\right)+1\right] \sum_{i=1}^{n} w_{i} x_{i} \\
& \leq \frac{1}{2}(M-m) \sum_{i=1}^{n} w_{i}\left|\ln x_{i}-\sum_{j=1}^{n} w_{j} \ln x_{i}\right| \\
& \leq \frac{1}{2}(M-m)\left[\sum_{i=1}^{n} w_{i}\left[\ln \left(x_{i}\right)+1\right]^{2}-\left(\sum_{i=1}^{n} w_{i}\left[\ln \left(x_{i}\right)+1\right]\right)^{2}\right]^{1 / 2}
\end{aligned}
$$

for any $w_{i} \geq 0(i=1, \ldots, n)$ with $\sum_{i=1}^{n} w_{i}=1$ and $x_{i} \in[m, M] \subset(0, \infty)(i=1, \ldots, n)$.

Now, if we let $m \rightarrow 0+$ and $M=1$ in (3.24) we get

$$
0 \leq \sum_{i=1}^{n} w_{i} x_{i} \ln \left(x_{i}\right)-\sum_{i=1}^{n} w_{i} x_{i} \ln \left(\sum_{i=1}^{n} w_{i} x_{i}\right)
$$




$$
\begin{aligned}
& \leq \sum_{i=1}^{n} w_{i}\left[\ln \left(x_{i}\right)+1\right] x_{i}-\sum_{i=1}^{n} w_{i}\left[\ln \left(x_{i}\right)+1\right] \sum_{i=1}^{n} w_{i} x_{i} \\
& \leq \frac{1}{2} \sum_{i=1}^{n} w_{i}\left|\ln x_{i}-\sum_{j=1}^{n} w_{j} \ln x_{i}\right| \\
& \leq \frac{1}{2}\left[\sum_{i=1}^{n} w_{i}\left[\ln \left(x_{i}\right)+1\right]^{2}-\left(\sum_{i=1}^{n} w_{i}\left[\ln \left(x_{i}\right)+1\right]\right)^{2}\right]^{1 / 2}
\end{aligned}
$$

for any $w_{i} \geq 0(i=1, \ldots, n)$ with $\sum_{i=1}^{n} w_{i}=1$ and $x_{i} \in(0,1](i=1, \ldots, n)$.

If $0<x \leq 1$, then $0<x^{p} \leq 1$ and if we apply the inequality (3.25) for $x_{j}=\left(x^{p}\right)^{j} \in(0,1]$, we have

$$
\begin{aligned}
0 \leq & \frac{p \ln x}{\sum_{j=0}^{k} a_{j} \alpha^{j}} \sum_{j=0}^{k} j a_{j} \alpha^{j}\left(x^{p}\right)^{j}-\frac{1}{\sum_{j=0}^{k} a_{j} \alpha^{j}} \sum_{j=0}^{k} a_{j} \alpha^{j}\left(x^{p}\right)^{j} \ln \left(\frac{1}{\sum_{j=0}^{k} a_{j} \alpha^{j}} \sum_{j=0}^{k} a_{j} \alpha^{j}\left(x^{p}\right)^{j}\right) \\
\leq & \frac{1}{\sum_{j=0}^{k} a_{j} \alpha^{j}}\left[p \ln x \sum_{j=0}^{k} j a_{j} \alpha^{j}\left(x^{p}\right)^{j}+\sum_{j=0}^{k} a_{j} \alpha^{j}\left(x^{p}\right)^{j}\right] \\
& -\frac{1}{\sum_{j=0}^{k} a_{j} \alpha^{j}}\left[p \ln x \sum_{j=0}^{k} j a_{j} \alpha^{j}+\sum_{j=0}^{k} a_{j} \alpha^{j}\right] \frac{1}{\sum_{j=0}^{k} a_{j} \alpha^{j}} \sum_{j=0}^{k} a_{j} \alpha^{j}\left(x^{p}\right)^{j} \\
\leq & \frac{1}{2}\left[\frac{1}{\sum_{j=0}^{k} a_{j} \alpha^{j}} \sum_{j=0}^{k} a_{j} \alpha^{j}[j p \ln x+1]^{2}-\left(\frac{1}{\sum_{j=0}^{k} a_{j} \alpha^{j}} \sum_{j=0}^{k} a_{j} \alpha^{j}[j p \ln x+1]\right)^{2}\right]^{1 / 2} \\
= & \frac{1}{2}\left[\frac{1}{\sum_{j=0}^{k} a_{j} \alpha^{j}} \sum_{j=0}^{k} a_{j} \alpha^{j}\left(j^{2} p^{2}(\ln x)^{2}+2 j p \ln x\right)+1-\left(\frac{p \ln x}{\sum_{j=0}^{k} a_{j} \alpha^{j}} \sum_{j=0}^{k} j a_{j} \alpha^{j}+1\right)^{2}\right]^{1 / 2} \\
= & \frac{1}{2}\left[\frac{p^{2}(\ln x)^{2}}{\sum_{j=0}^{k} a_{j} \alpha^{j}} \sum_{j=0}^{k} j^{2} a_{j} \alpha^{j}+\frac{2 p \ln x}{\sum_{j=0}^{k} a_{j} \alpha^{j}} \sum_{j=0}^{k} j a_{j} \alpha^{j}+1-\left(\frac{p \ln x}{\sum_{j=0}^{k} a_{j} \alpha^{j}} \sum_{j=0}^{k} j a_{j} \alpha^{j}+1\right)^{2}\right]^{1 / 2} \\
= & \left.\frac{1}{2} p|\ln x| \frac{1}{\sum_{j=0}^{k} a_{j} \alpha^{j}} \sum_{j=0}^{k} j^{2} a_{j} \alpha^{j}-\left(\frac{1}{\sum_{j=0}^{k} a_{j} \alpha^{j}} \sum_{j=0}^{k} j a_{j} \alpha^{j}\right)^{2}\right]^{1 / 2} \cdot
\end{aligned}
$$

Since all series whose partial sums involved in the inequality (3.26) are convergent, then by letting $k \rightarrow \infty$ in (3.26) we deduce (3.21).

Corollary 3. Let $f(z)=\sum_{n=0}^{\infty} a_{n} z^{n}$ be a power series with nonnegative coefficients and convergent on the open disk $D(0, R)$ with $R>0$ or $R=\infty$. If $p>1, \frac{1}{p}+\frac{1}{q}=1$ and $u, v>0$ with $v^{p} \leq u^{q}<R$, then

$$
\begin{aligned}
0 & \leq \frac{f\left(v^{p}\right)}{f\left(u^{q}\right)} \ln \left(\frac{f\left(u^{q}\right)}{f\left(v^{p}\right)}\right)-\frac{v^{p} f^{\prime}\left(v^{p}\right)}{f\left(u^{q}\right)} \ln \left(\frac{u^{q}}{v^{p}}\right) \\
& \leq u^{q}\left[\frac{f\left(v^{p}\right)}{f\left(u^{q}\right)} \frac{f^{\prime}\left(u^{q}\right)}{f\left(u^{q}\right)}-\frac{\frac{v^{p}}{u^{q}} f^{\prime}\left(v^{p}\right)}{f\left(u^{q}\right)}\right] \ln \left(\frac{u^{q}}{v^{p}}\right)
\end{aligned}
$$




$$
\leq \frac{1}{2}\left[\frac{u^{q}\left[f^{\prime}\left(u^{q}\right)+u^{q} f^{\prime \prime}\left(u^{q}\right)\right]}{f\left(u^{q}\right)}-\left(\frac{u^{q} f^{\prime}\left(u^{q}\right)}{f\left(u^{q}\right)}\right)^{2}\right]^{1 / 2} \ln \left(\frac{u^{q}}{v^{p}}\right)
$$

\section{Example 4.}

(a) If we write the inequality (3.21) for the function $\frac{1}{1-z}=\sum_{n=0}^{\infty} z^{n}, z \in D(0,1)$, then we have for $\alpha, x \in(0,1)$ and $p>0$ that

$$
\begin{aligned}
0 & \leq \frac{p \alpha x^{p}(1-\alpha)}{\left(1-\alpha x^{p}\right)^{2}} \ln x-\frac{1-\alpha}{\left(1-\alpha x^{p}\right)} \ln \left(\frac{1-\alpha}{1-\alpha x^{p}}\right) \\
& \leq p \alpha\left[\frac{x^{p}(1-\alpha)}{\left(1-\alpha x^{p}\right)^{2}}-\frac{1}{1-\alpha x^{p}}\right] \ln x \leq \frac{1}{2} \frac{p \alpha^{1 / 2}}{1-\alpha}|\ln x| .
\end{aligned}
$$

(b) If we write the inequality (3.21) for the function $\exp z=\sum_{n=0}^{\infty} \frac{1}{n !} z^{n}, z \in \mathbb{C}$, then we have

$$
\begin{aligned}
0 & \leq\left[p \alpha x^{p} \ln x-\alpha\left(x^{p}-1\right)\right] \exp \left[\alpha\left(x^{p}-1\right)\right] \\
& \leq p \alpha\left(x^{p}-1\right) \exp \left[\alpha\left(x^{p}-1\right)\right] \ln x \leq \frac{1}{2} p|\ln x| \alpha^{1 / 2}
\end{aligned}
$$

for $x \in(0,1)$ and $\alpha, p>0$.

\section{Acknowledgement}

The author would like to thank the anonymous referee for valuable comments that have been implemented in the final version of the paper.

\section{References}

[1] P. Cerone and S. S. Dragomir, A refinement of the Grüss inequality and applications, Tamkang J. Math., 38(2007), 37-49. Preprint RGMIA Res. Rep. Coll., 5(2) (2002), Art. 14.

Online http://rgmia.org/papers/v5n2/RGIApp.pdf.

[2] P. Cerone and S. S. Dragomir, Some applications of de Bruijn's inequality for power series, Integral Transform. Spec. Funct. 18 (2007), 387-396.

[3] S. S. Dragomir, Discrete Inequalities of the Cauchy-Bunyakovsky-Schwarz Type, Nova Science Publishers Inc., N.Y., 2004.

[4] S. S. Dragomir and N. M. Ionescu, Some converse of Jensen's inequality and applications, Rev. Anal. Numér. Théor. Approx., 23 (1994), 71-78. MR1325895 (96c:26012).

[5] A. Ibrahim and S. S. Dragomir, Power series inequalities via Buzano's result and applications, Integral Transform. Spec. Funct., 22 (2011), 867-878.

[6] A. Ibrahim and S. S. Dragomir, Power series inequalities via a refinement of Schwarz inequality, Integral Transform. Spec. Funct., 23 (2012), 769-78.

[7] A. Ibrahim and S. S. Dragomir, A survey on Cauchy-Bunyakovsky-Schwarz inequality for power series, 247295, in G. V. Milovanović and M. T. Rassias (eds.), Analytic Number Theory, Approximation Theory, and Special Functions, Springer, 2013. DOI 10.1007/978-1-4939-0258-3_10,

[8] A. Ibrahim, S. S. Dragomir and M. Darus, Some inequalities for power series with applications, Integral Transform. Spec. Funct., 24 (2013), 364-376. 
[9] A. Ibrahim, S. S. Dragomir and M. Darus, Power series inequalities related to Young's inequality and applications, Integral Transforms Spec. Funct., 24 (2013), 700-714.

[10] A. Ibrahim, S. S. Dragomir and M. Darus, Power series inequalities via Young's inequality with applications, J. Inequal. Appl. 2013, 2013:314, 13 pp.

Mathematics, School of Engineering \& Science, Victoria University, PO Box 14428, Melbourne City, MC 8001, Australia.

School of Computational \& Applied Mathematics, University of the Witwatersrand, Private Bag 3, Johannesburg 2050, South Africa.

E-mail: sever.dragomir@vu.edu.au 